\title{
The impact of mesenchymal stem cells on host immunity and disease outcome in bacterial lung infection
}

\author{
Authors: Janet Stowell, ${ }^{A}$ Catherine Reynolds ${ }^{B}$ and Rosemary Boyton ${ }^{B}$
}

\section{Introduction}

Bacterial pneumonia is the most common infectious cause of death worldwide, disease burden is increasing and antibiotic resistance continues to escalate. ${ }^{1,2}$ Novel therapies are needed. Mesenchymal stem cells (MSCs) are a heterogeneous subset of stromal stem cells which reside in bone marrow and other anatomic niches. ${ }^{3,4}$ MSCs have been demonstrated to regulate lung inflammation and enhance bacterial clearance in preclinical models of pneumonia and sepsis, making them an attractive novel treatment for bacterial lung infection resistant to antibiotic therapy. ${ }^{5-7}$ There remain many unanswered questions about the mechanisms by which MSCs achieve their immunomodulatory effects, particularly relating to MSC crosstalk with immune effector cells from both the innate and adaptive immune systems. A putative mechanism by which MSCs have anti-inflammatory effects is via the recruitment of regulatory T cells (Tregs). 8,9

Tregs are a subset of $\mathrm{CD} 4^{+} \mathrm{T}$ cells acknowledged to be important in maintaining the fine balance of immune responses during bacterial infection. ${ }^{10}$ Their precise role in streptococcal and pseudomonal lung infection is undefined and their interaction with MSCs in the context of bacterial infection is undetermined. This project explores the impact of MSCs on host immunity in bacterial lung infection. This involves an investigation of MSC antimicrobial effect in established experimental mouse models of Gram-positive and Gram-negative bacterial lung infection and exploration of the potential role of Tregs contributing to the observed antimicrobial effects.

\section{Materials and methods}

Autologous murine MSCs derived from compact bone and bone marrow are used in mouse models of Streptococcus pneumoniae and Pseudomonas aeruginosa pneumonia. MSC treatment is given intravenously 3 hours into a 24 -hour infection. All mice are on a C57Bl/6 background, including the Foxp3-DTR inducible Treg knockout utilised to investigate the effect of Treg depletion on MSC function. This mouse has a targeting construct encoding human diphtheria toxin receptor fused to

Authors: ${ }^{\mathrm{A}}$ Royal Brompton Hospital, London, UK; ${ }^{\mathrm{B}}$ Imperial College London, UK sequences encoding green fluorescent protein (GFP) inserted into the Foxp3 gene. Exposure to diphtheria toxin leads to depletion of Tregs. Infection and MSC treatment studies are carried out with and without Tregs.

\section{Results and discussion}

Systemic treatment with MSCs reduced bacterial burden in lung and bronchoalveolar lavage (BAL) following intranasal infection with $S$ pneumoniae and $P$ aeruginosa. Levels of pro-inflammatory cytokines in lung (TNF- $\alpha$, IL-6, IFN- $\gamma$ and IL-17F) and BAL (IL-6) were also significantly reduced. Depletion of Tregs prior to infection with $S$ pneumoniae resulted in a significant increase in lung bacterial burden. In contrast, Treg depletion during $P$ aeruginosa lung infection did not impact on bacterial burden or inflammatory response. Following infection with $S$ pneumoniae, bacterial burden and inflammatory response were greater in MSC-treated Tregdepleted mice than in the Treg-replete cohort. In contrast, in the $P$ aeruginosa lung infection model, MSC protective effect was preserved following Treg depletion.

\section{Conclusion}

MSCs may be utilised for the treatment of pneumonia. Tregs are beneficial to the host response to $S$ pneumoniae, but have no role in the host response to $P$ aeruginosa. MSC protective effect for $S$ pneumoniae appears to be partially Treg dependent. For $P$ aeruginosa, MSC protective effect is independent of Tregs.

\section{Conflicts of interest}

None declared.

\section{References}

1 Ramirez JA, Anzueto AR. Changing needs of community-acquired pneumonia. J Antimicrob Chemother 2011;66 Suppl 3:iii3-9.

2 Kim L, McGee L, Tomczyk S, Beall B. Biological and epidemiological features of antibiotic-resistant Streptococcuspneumoniae in pre- and postconjugate vaccine eras: a United States perspective. Clin Microbiol Rev 2016;29:525-52.

3 Dominici M, Le Blanc K, Mueller I et al. Minimal criteria for defining multipotent mesenchymal stromal cells. The International Society for Cellular Therapy position statement. Cytotherapy 2006;8:315-7. 
4 da Silva Meirelles L, Chagastelles PC, Nardi NB. Mesenchymal stem cells reside in virtually all post-natal organs and tissues. J Cell Sci 2006;119:2204-13.

5 Mei SHJ, Haitsma JJ, Dos Santos CC et al. Mesenchymal stem cells reduce inflammation while enhancing bacterial clearance and improving survival in sepsis. Am J Respir Crit Care Med 2010;182:1047-57.

6 Zhang L, Gallo RL. Antimicrobial peptides. Curr Biol 2016;26:R14-9.

7 Park J, Kim S, Lim H et al. Therapeutic effects of human mesenchymal stem cell microvesicles in an ex vivo perfused human lung injured with severe E. coli pneumonia. Thorax 2019;74:43-50.
8 Nemeth K, Keane-Myers A, Brown JM et al. Bone marrow stromal cells use TGF- $\beta$ to suppress allergic responses in a mouse model of ragweed-induced asthma. Proc Natl Acad Sci U S A 2010;107:5652-7.

9 Lee HJ, Kim SN, Jeon MS, Yi T, Song SU. ICOSL expression in human bone marrow-derived mesenchymal stem cells promotes induction of regulatory T cells. Sci Rep 2017;7:44486.

10 Neill DR, Fernandes VE, Wisby L et al. T regulatory cells control susceptibility to invasive pneumococcal pneumonia in mice. PLoS Pathog 2012;8:e1002660. 